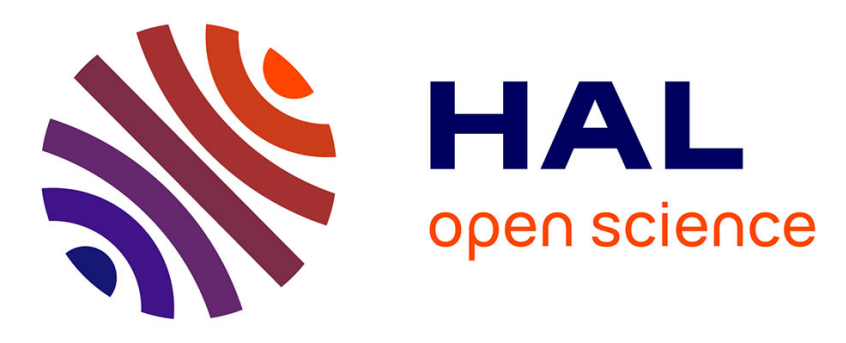

\title{
A virtual assistant dedicated to supporting day-to-day medical consultations
}

\author{
Antoine Richard, Brice Mayag, François Talbot, Alexis Tsoukias, Yves \\ Meinard
}

\section{- To cite this version:}

Antoine Richard, Brice Mayag, François Talbot, Alexis Tsoukias, Yves Meinard. A virtual assistant dedicated to supporting day-to-day medical consultations. 2021 IEEE 9th International Conference on Healthcare Informatics (ICHI), Aug 2021, Victoria, Canada. pp.330-338, 10.1109/ICHI52183.2021.00057 . hal-03381829

\section{HAL Id: hal-03381829 \\ https://hal.archives-ouvertes.fr/hal-03381829}

Submitted on 18 Oct 2021

HAL is a multi-disciplinary open access archive for the deposit and dissemination of scientific research documents, whether they are published or not. The documents may come from teaching and research institutions in France or abroad, or from public or private research centers.
L'archive ouverte pluridisciplinaire HAL, est destinée au dépôt et à la diffusion de documents scientifiques de niveau recherche, publiés ou non, émanant des établissements d'enseignement et de recherche français ou étrangers, des laboratoires publics ou privés. 


\title{
A virtual assistant dedicated to supporting day-to-day medical consultations
}

\author{
Antoine Richard* ${ }^{* \ddagger}$ Brice Mayag*, François Talbot ${ }^{\dagger}$, Alexis Tsoukias* and Yves Meinard* \\ *Université Paris-Dauphine, PSL Research University, CNRS, LAMSADE, UMR 7243, 75016 Paris, France \\ ${ }^{\dagger}$ DSII Bron, Hospices Civils de Lyon, 61 Boulevard Pinel, 69672 Bron, France \\ ${ }^{\ddagger}$ GIE Hopsis Lyon, 3 Quai de Célestins, 69229 Lyon, France
}

\begin{abstract}
In this paper, we present a virtual assistant developed in collaboration with the civil hospitals of Lyon ("Hospices Civils de Lyon" or HCL in french), a group of hospitals in the area of Lyon (France). This virtual assistant is dedicated to supporting physicians during day-to-day medical consultations. It aims to anticipate which pieces of information physicians will need, given the information already known on patients, and provide them with these needed pieces of information. According to clinical trials made at the HCL, physicians appreciate the help that the proposed system provides for day-to-day practices.
\end{abstract}

Index Terms-Clinical Decision Support System, Virtual Assistant, Health Informatics

\section{INTRODUCTION}

In collaboration with the civil hospitals of Lyon ("Hospices Civils de Lyon" or HCL in French), a group of 14 hospitals in the area of Lyon (France), we have developed a virtual assistant, called CoBoy, dedicated to supporting day-to-day medical consultations. This virtual assistant was developed as a module of the Health Information System (HIS) currently used by HCL's clinicians: Easily ${ }^{\circledR}$.

Consultations are a large part of the HCL's physicians' activities (they performed 1 million of them in 2020). During these consultations, physicians make decisions repeatedly and we aim to support physicians during these customary activities. Diagnostic Decision Support Systems (DDSSs) have been developed as an attempt to support physicians during diagnosis decisions [1], [2].

Famous examples of DDSSs include MYCIN [3], [4], an expert system dedicated to antimicrobial therapy, or INTERNIST-1/QMR [5], [6], an expert system designed to support decisions in generalist medicine. The CHICA system, used at Wishard Memorial Hospital of Indianapolis, is a more recent example dedicated to supporting child health in primary care [7]-[9]. The eIMCI and ALMANACH projects are also recent examples of DDSSs dedicated to supporting child health in developing countries [10], [11].

Some systems based on machine learning algorithms, also called ML-based DDSSs, have recently been developed to support diagnoses. These ML-based DDSSs include, for example, systems dedicated to detecting ocular diseases from a picture of a patient's eye [12]-[14] or systems dedicated to detecting cancer nodules on radiographies [15]-[18].

However, a well-known problem of DDSSs is their lack of acceptability by physicians during day-to-day practices and non-complex situations, which leads physicians to overriding or ignoring current DDSSs' recommendations [19][21]. These recommendations are generally "gold-standard" guidelines or suggestions of diagnoses. In previous works [22], we have argued that this lack of acceptability is due to the fact that the support provided by current DDSSs is at odds with the constraints implied by supporting decisions during day-to-day practices and non-complex situations, like customary medical consultations. The main constraint that a DDSS have to deal with, in such situations, is the strong will of the physicians to stay in charge of their decision processes and to stay responsible of the safety of their patient. We concluded that an adapted approach for decision support during customary medical consultations must respect the know-how of physicians and leave them the responsibility of the decisions taken during these consultations, by limiting itself to providing what physicians need most during consultations: pieces of information concerning their patients [22], [23].

Accordingly, we have developed a decision support system, in the form of a virtual assistant, able to anticipate and provide pieces of information that the physician needs to reach diagnoses concerning a specific patient. This system aims to reduce the workload of physicians and to allow them to make informed decisions during medical consultations. In section II, we present the system's capabilities: how it determines which pieces of information to provide to physicians using both a rule-based classifier and a Naive Bayes classifier, how it searches for raw data associated to these pieces of information in the database of Easily ${ }^{\circledR}$, and how it displays these pieces of information to physicians through its user interface. In section III, we present a simple use case scenario to illustrate the process of the system during a medical consultation. In section IV, we present the clinical trials we made with physicians of the HCL's department of endocrinology. As a result of these clinical trials, despite some practical limitations, physicians showed high acceptability and much enthusiasm for the proposed system. Lastly, in section $\mathrm{V}$, we discuss limitations and perspectives, before briefly concluding in section VI.

\section{THE PROCESS OF THE PROPOSED SYSTEM}

As introduced in section I, the goal of CoBoy is to determine which pieces of information on a patient could be needed by a physician for a specific consultation, to search for raw data associated to each of these pieces of information in the 
database of Easily ${ }^{\circledR}$, and to display these pieces of information to physicians in an interpretable way. Fig. 1 summarizes the process of CoBoy at the beginning of a medical consultation.

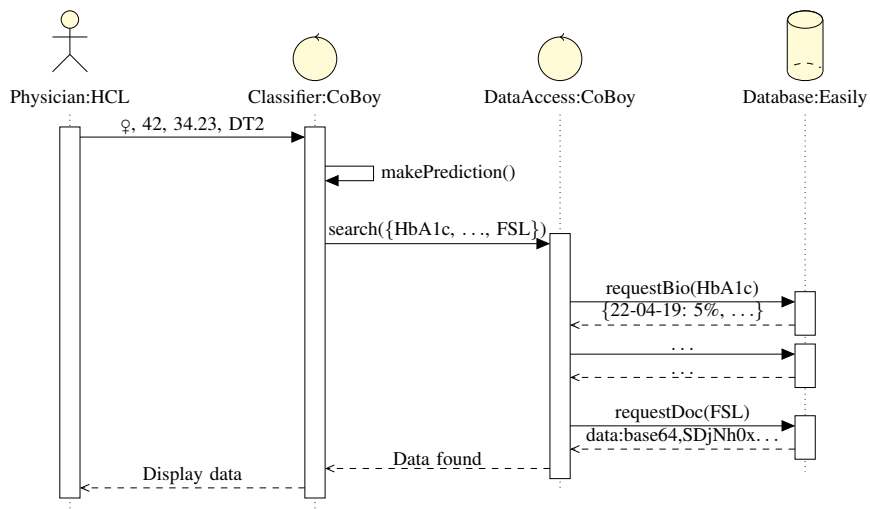

Fig. 1. Summary of the CoBoy's process when providing pieces of information on a patient to a physician at the beginning of a medical consultation

In this section, we detail the different steps of this process. Sub-section II-A details how the system determines, based on two classification systems, which pieces of information are needed by a physician for a specific consultation. Subsection II-B then explains how the system searches for raw data associated to each piece of information needed, given their data type. Lastly, sub-section II-C details how pieces of information collected by the system are displayed to users.

\section{A. Determining which pieces of information on patients are needed}

The use case of determining which pieces of information physicians need for specific consultations can be formalized as a multi-label classification problem [24]. Sex, age, BMI, and the pathology of the patient are common pieces of information about patients that physicians always have at the beginning of consultations. The learning algorithm will use these pieces of information as a base $\mathcal{X}$ to learn which other pieces of information on a patient $\mathcal{Y}$ are needed and which pieces of information are not. Each label corresponds to each piece of information on a patient that could be needed by a physician during a medical consultation. Table I shows an example, with fictitious data, of our use case.

TABLE I

EXAMPLE OF A MULTI-LABEL DATASET BASED ON OUR USE CASE

\begin{tabular}{|c|c|c|c|c|c|c|c|c|c|}
\hline \multicolumn{4}{|c|}{$\mathcal{X}$ : pieces of information known on patients } & \multicolumn{6}{|c|}{$\mathcal{Y}$ : pieces of information on patients needed by physicians } \\
\hline Sex & Age & BMI & Disease & 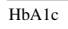 & Blood Sugar & HDL & LDL & Creatinine & Microalbumin \\
\hline $9=$ & $\begin{array}{ll}42 \\
52\end{array}$ & 34.23 & $\begin{array}{l}\text { DT2 } \\
H C h o l\end{array}$ & 1 & 1 & 0 & 0 & 0 & 0 \\
\hline 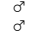 & 24 & $\begin{array}{l}27.15 \\
21.12\end{array}$ & $\begin{array}{l}\text { HChol } \\
\text { DT1 }\end{array}$ & $\begin{array}{l}0 \\
1\end{array}$ & $\begin{array}{l}0 \\
1\end{array}$ & ${ }_{0}^{1}$ & $\begin{array}{l}1 \\
0\end{array}$ & $\begin{array}{l}0 \\
1\end{array}$ & $\begin{array}{l}0 \\
1\end{array}$ \\
\hline q & & 26.22 & HChol & 0 & 0 & 1 & 1 & 0 & 0 \\
\hline
\end{tabular}

To tackle this problem, we've developed a version of the well-known Naive Bayes algorithm [25] adapted to multi-label problems. According to previous works on algorithmic transparency [26], the Naive Bayes algorithm is one of the easiest learning algorithms understandable by physicians. The choice to search for transparency is motivated by the results of studies that have shown that transparent support systems are more accepted in practices by physicians, including those that are more recalcitrant to support systems [27], [28]. Transparency also potentially allows a better understanding of a DDSS and then a better use of this DDSS. Besides, for use cases without complex correlations to learn, like ours, it is possible to use a transparent algorithm without fearing losing performance [26].

To determine which pieces of information are probably needed by a physician for a specific consultation with a patient, CoBoy computes an estimation of the probability $P\left(Y=1 \mid X_{p}\right)$ that a piece of information $Y$ could be needed by the physician, with $X_{p} \in \mathcal{X}$ a set of pieces of information known on the patient at the beginning of the consultation. To do so, CoBoy uses a set of probabilities learned from logs of pieces of information searched or recorded by physicians during previous consultations, and applies a naive version of the Bayes theorem (1) which assumes that each variable $X \in \mathcal{X}$ are independent of each other.

$$
P\left(Y=1 \mid X_{p}\right)=\frac{P(Y=1) \prod_{X \in \mathcal{X}} P(X=x \mid Y=1)}{\sum_{y=0}^{1}\left(P(Y=y) \prod_{X \in \mathcal{X}} P(X=x \mid Y=y)\right)}
$$

Therefore, if $P\left(Y=1 \mid X_{p}\right) \geq \lambda$ the label $Y \in \mathcal{Y}$ is set to 1 , meaning that the physician will probably need the piece of information $Y$ on the current patient $p$. The $\lambda$ parameter is used as a threshold to define from which minimum probability a piece of information on a patient may be needed by a physician. By default, this threshold $\lambda$ is set at 0.5 .

However, the current granularity of logs recorded by Easily ${ }^{\circledR}$ for some pieces of information searched by physicians during their consultations limits the construction of a precise learning dataset. We have therefore decided to complete the Naive Bayes classification algorithm with a classification system based on rules defined by physicians of the HCL themselves. A rule-based system is still transparent for physicians [26], [29], especially if rules are defined by physicians themselves and not determined by a learning algorithm. These rules take the form of "IF ... THEN ..." implications, for example:

\section{IF disease $=$ diabetes THEN SEARCH FOR: HbA1c}

Thereby obtained a mixed classification system to determine which pieces of information are needed by a physician for a specific situation. When a physician asks CoBoy for pieces of information on a given patient, the system will use the set of rules defined by physicians to determine a first set of pieces of information to provide, and then it will use the Naive Bayes classification system to complete this set.

Currently, the CoBoy system is able to search for, depending on physicians' needs, close to 50 types of biological analyses and close to 30 types of medical documents. Besides, CoBoy is currently able to support consultations for around twenty different kinds of diseases. 


\section{B. Searching for raw data in the database}

Once the pieces of information to provide to the physician are determined for a specific medical consultation, the system searches for raw data associated to these pieces of information into the database Easily ${ }^{\circledR}$. For each data type, CoBoy will request the HCL's database in a specific way to obtain the targeted data.

For biological analyses made in medical laboratories, this is the result of the last analysis and the results history that are requested. Because biological analyses are often linked to minimum and maximum thresholds, the system also searches for these thresholds if they are available. Let us specify that results of analyses made by patients in medical laboratories outside the HCL are not available into the database of Easily ${ }^{\circledR}$ if the laboratory has not transmitted these results to the HCL. Due to this practical limitation, CoBoy is unable to provide these results to physicians. For performance reasons, only the hundred most recent results are requested.

For medical documents, such as reports of medical imaging (scanners, MRI, radiographies, etc.), reports from other specialists following the current patient (ophtalmology, dietetic, cancerology, etc.), or drug prescriptions, CoBoy searches for the most recent document in the database.

For "general" pieces of information, such as background history, family medical history, or patient's allergies, CoBoy searches for all data about it into the database of Easily ${ }^{\circledR}$.

Therefore, CoBoy collects into the database of Easily ${ }^{\circledR}$ all the raw data associated to pieces of information that could be needed by a physician for the current patient, according to the subset determined before (see sub-section II-A). However, it could happen that CoBoy does not find any data concerning the current patient for some pieces of information requested. In such cases, CoBoy simply returns the fact that no data was found in the database for these pieces of information, because the absence of data on the current patient can also be relevant to the decision process of physicians [23].

\section{Displaying pieces of information to physicians}

CoBoy then displays all the targeted pieces of information on the patient to the physician according to their type. Fig. 2 displays, with fictitious data, the current user interface of CoBoy.

This user interface is divided into two columns. The left column is dedicated to displaying the results of biological analyses and "general" pieces of information. For each piece of information corresponding to biological analyses, CoBoy plots the results history and highlights the latest result obtained. If biological analyses were associated with thresholds, CoBoy also plots these thresholds with red horizontal lines. Concerning "general" pieces of information, CoBoy simply displays them textually.

The right column is dedicated to displaying medical documents concerning the current patient. These documents are displayed using a PDF viewer and are accessible through a tab system.
Pieces of information determined using the Naive Bayes classifier (see sub-section II-A) are sorted according to the estimated probability that the physician will need them during the consultation. The more probable is the higher, but pieces of information determined by the rule-based system come first. In the case in which no data was found in the HCL's database for a piece of information, CoBoy says that it has searched for raw data for these piece of information but didn't found anything, with a message such as "I didn't find any data about HbA1c of this patient".

1) Results' explanations: Each provided piece of information is associated with explanations of how CoBoy determined that the physician could need this piece of information during the current consultation. These explanations are available by clicking on the information point next to the associated name of the piece of information (see Fig. 2).

For pieces of information determined using the Naive Bayes classifier, two levels of explanations are provided. First, CoBoy provides the probability that the physician may need this piece of information on the patient given the patient's age, weight, BMI, and disease. This first level of explanation takes the form of a sentence such as: "Based on your previous consultations and given the sex, age, BMI, and disease of the patient, the probability that you may need to know her/his HbAlc is $72 \%$ ". The second level of explanation, accessible by clicking on a "Details" button, contains the details of the calculations made to produce this result. This second level of explanation allows the physician to know which pieces of information were crucial in the prediction of her/his needs.

For pieces of information determined using the rule-based system, CoBoy provides the activation condition of the rule. This explanation takes the form of a sentence such as: "The $\mathrm{HbAlc}$ of the current patient is provided because: disease $=$ diabetes".

There are also pieces of information on patients that are requested and displayed by default for all the patients, such as the report of the last consultation or her/his last drug prescription. In this case, as an explanation, CoBoy simply says that this piece of information on the patient is requested by default.

2) User's feedbacks: In addition, each piece of information provided by CoBoy is associated with a switch button (see Fig. 2). This button allows the physician to indicate to CoBoy if s/he didn't need the associated piece of information for the current patient. This feedback feature allows us to have an estimation of CoBoy's precision in practical use cases.

The feedback button is also available for pieces of information requested by CoBoy but not found in databases. The goal is to know whether or not CoBoy was right to request a specific piece of information for a specific medical consultation.

In the case of pieces of information on patients displayed by default, this feature is not available, because default pieces of information do not depend on CoBoy's predictions. 


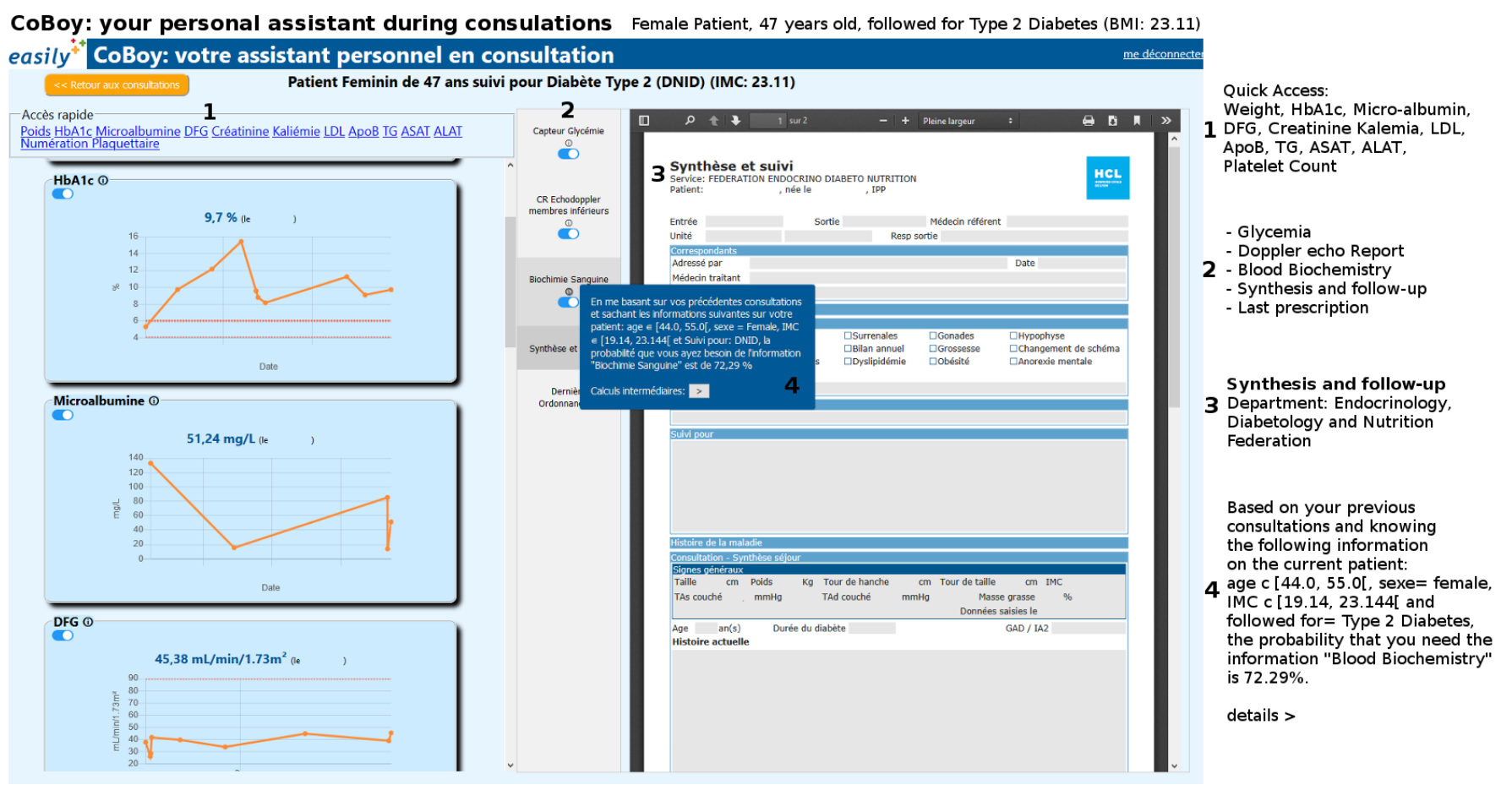

Fig. 2. The current user interface of CoBoy (with fictitious data)

\section{USE CASE}

In this section, we use a simplified example of a medical consultation to illustrate the process of CoBoy. Let us take a fictitious patient $p$ characterized by the set $X_{p}=\{$ sex : o, age : $42, b m i$ : 34.23 ,disease : $D T 2\}$, with $D T 2$ corresponding to type 2 diabetes. At the beginning of the consultation, the physician selects the patient in her/his list of patients for the day.

When the patient is selected, CoBoy uses its rule-based classifier to determine a first subset of pieces of information to provide to the physician. Let us suppose that this only rule is activated: "IF disease=diabetes THEN SEARCH FOR HbAlc", because the patient $p$ is followed up for her/his type 2 diabetes.

Then CoBoy computes the probabilities that the physician may need additional pieces of information on the patient during the consultation. As developed in sub-section II-A, these probabilities are computed by using the Naive Bayes classifier of CoBoy, given the set $X_{p}$ of common information on the patient. For each piece of information $Y \in \mathcal{Y}$ potentially needed by physicians, CoBoy computes the probability $P(Y=1 \mid X)$ by applying Bayes' theorem (1). Let us suppose that pieces of information potentially needed by physicians are glycated hemoglobin (HbA1c), blood sugar, HDL cholesterol, LDL cholesterol, creatinine, and microalbumin. Because "HbA1c" is already determined as a piece of information to provide to the physician, CoBoy will not compute the probability that the physician may need "HbA1c" for the current patient $p$. Let us suppose that CoBoy computes the following probabilities (2).

$$
\begin{gathered}
P\left(\text { Blood Sugar }=1 \mid X_{p}\right) \simeq 0.66 \\
P\left(\mathrm{HDL}=1 \mid X_{p}\right) \simeq 0.01 \\
P\left(\mathrm{LDL}=1 \mid X_{p}\right) \simeq 0.01 \\
P\left(\text { Creatinine }=1 \mid X_{p}\right) \simeq 0.34 \\
P\left(\text { Microalbumin }=1 \mid X_{p}\right) \simeq 0.42
\end{gathered}
$$

In this example, if we use a threshold $\lambda=0.5$, CoBoy determines that the physician probably needs to obtain the patient's blood sugar history. We can see in this example that, with a more permissive threshold, e.g. $\lambda=0.4$, CoBoy would also determine that the physician probably needs to know the microalbumin history of the patient. However, using a more permissive threshold may overwhelm physicians with useless pieces of information on the patient.

Once CoBoy has determined which pieces of information to provide to the physician, it requests associated raw data in the HCL's database. In our example, CoBoy has to request raw data for glycated hemoglobin (HbA1c) and blood glucose history concerning the current patient.

The HbAlc is a piece of information obtained through laboratory analyses. It is hence a biological analysis on the patient. CoBoy then requests the hundred most recent results concerning the patient's HbAlc from the HCL's database. Once it has obtained these data, CoBoy plots the results history and displays it to the physician (see Fig. 2).

Concerning the patient's blood sugar history, this piece of information can also be considered to be a biological analysis. 
However, blood sugar is generally collected by patients themselves using a blood sugar meter. CoBoy then requests the document produced by the patient's blood sugar meter and displays it to the physician (see Fig. 2). If the patient doesn't have a blood sugar meter, nothing is displayed to the physician, but CoBoy tells the physician that the piece of information has been searched but not found.

\section{Clinical TRials}

In this section, we detail the clinical trials we have conducted at the HCL to evaluate the viability and the applicability of our decision support system during real consultations. Sub-section IV-A presents our observation protocol and our hypotheses. Sub-section IV-B then details the results obtained from our clinical trials.

\section{A. Protocol}

We use here the term "observation" to refer to the study of one, and only one, consultation starting when the patient enters the consultation room and ending when s/he leaves it. We use the term "observation session" to denote a set of consecutive observations. Fig. 3 schematizes the setting during observations. A second screen has been used to allow physicians to access to both Easily ${ }^{\circledR}$ and CoBoy at the same time.

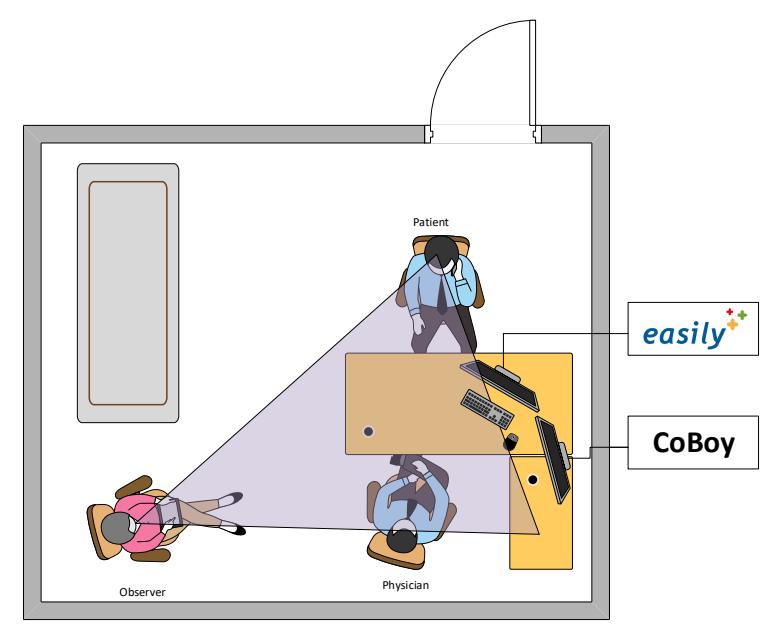

Fig. 3. Agents' disposition during clinical trials of CoBoy

The first part of this study aims to evaluate whether the number of pieces of information provided by the systems had an impact, beneficial or not, on physicians' decision processes. During observations, the following criteria have been monitored by the observer:

- The number of pieces of information provided by CoBoy, in order to quantify the support provided by the system;

- The number of pieces of information searched by the physician, explained orally to the patient, in order to quantify the need for information of the physician;

- The number of mouse clicks made by the physician when using Easily ${ }^{\circledR}$, used to quantify physician-HIS interactions;
- The duration of the consultation.

We hypothesize that, if pieces of information provided by our decision support system are useful to physicians, this will decrease the need for physicians to look for other pieces of information on their own. In other words, the higher the number of pieces of information provided, the lower the number of pieces of information searched by physicians on their own. We have chosen to use the number of mouse clicks made by physicians, when they use Easily ${ }^{\circledR}$, as an indicator of their interactions with their HIS when they search for or record pieces of information. Hence, according to our assumptions, the higher the number of pieces of information provided, the lower the number of mouse clicks. Concerning the duration of consultations, physicians generally try to stay on schedule as much as possible. Therefore, we assume that the number of pieces of information provided by our system will not impact, either positively or negatively, the duration of consultations.

However, the introduction of a new tool in physicians' work processes unavoidably entails an entry cost [22], [30]. Therefore, we expect to observe, in many cases, a rise in the number of pieces of information searched, the number of mouse clicks, and the duration of consultations, due to the introduction of CoBoy in the work processes of physicians. If the correlation between the rise of these three criteria and the rise of the number of pieces of information provided by the tool is weak, overall this will support the hypothesis that the support provided by CoBoy has been able to compensate this inevitable entry cost.

The second part of our study aims to assess the potential acceptability of the developed decision support system. To that end, we asked physicians to fill in a brief questionnaire at the end of observation sessions, once all the scheduled consultations were finished. To minimize the impact of the presence of the observer on physicians' answers, questionnaires were completed anonymously and were then shuffled. As a consequence, the two parts of our study cannot be connected. This questionnaire is composed of three main questions, aiming to assess respectively the useability of the system, the perceived utility of the system, and the intention to use the system in day-to-day practices:

1) "Would you say that getting started with CoBoy is":

- "Very easy"

- "Rather easy"

- "Neither easy nor difficult"

- "Rather difficult"

- "Very difficult"

2) "Would you say that, during consultations, CoBoy is":

- "Very useful"

- "Rather useful"

- "Neither useful nor useless"

- "Rather useless"

- "Totally useless"

3) "If the HCL integrated CoBoy into Easily ${ }^{\circledR}$, would you use CoBoy during your consultations?"

- "Certainly" 
- "Rather yes"

- "I don't know"

- "Rather no"

- "Certainly not"

For each question, physicians were given the opportunity to complete their answers with "additional comments". Finally, we asked two general questions to physicians concerning their opinion about the decision support system and potential improvements:

- "Do you have general comments concerning CoBoy?"

- "Do you have suggestions for improvements?"

\section{B. Results}

Overall, we have observed 49 consultations performed by 7 physicians working at the HCL's department of endocrinology and using Easily ${ }^{\circledR}$ every day. 4 to 11 consultations were observed for each physician, with a mean of 7 observations per physician. Table II summarizes, for each observed physician, the means and standard deviations of results for each criterion observed during clinical trials.

TABLE II

SUMMARY OF RESULTS OBTAINED FOR EACH PHYSICIAN OBSERVED DURING CLINICAL TRIALS OF COBOY

\begin{tabular}{cccccc}
\hline Physician & $\begin{array}{c}\text { Number of } \\
\text { observations }\end{array}$ & $\begin{array}{c}\text { Mean number of } \\
\text { pieces of information } \\
\text { provided }\end{array}$ & $\begin{array}{c}\text { Mean number of } \\
\text { pieces of information } \\
\text { searched }\end{array}$ & $\begin{array}{c}\text { Mean number of } \\
\text { mouse clicks }\end{array}$ & $\begin{array}{c}\text { Mean duration } \\
\text { of consultations } \\
\text { (in minutes) }\end{array}$ \\
\hline 1 & 8 & $14 \pm 3$ & $14 \pm 2$ & $82 \pm 26$ & $23 \pm 2$ \\
2 & 6 & $11 \pm 5$ & $14 \pm 5$ & $126 \pm 44$ & $26 \pm 7$ \\
3 & 4 & $14 \pm 1$ & $15 \pm 2$ & $188 \pm 78$ & $35 \pm 11$ \\
4 & 11 & $8 \pm 6$ & $8 \pm 2$ & $96 \pm 32$ & $18 \pm 4$ \\
5 & 8 & $12 \pm 6$ & $19 \pm 5$ & $97 \pm 39$ & $23 \pm 6$ \\
6 & 6 & $15 \pm 1$ & $16 \pm 1$ & $208 \pm 69$ & $36 \pm 10$ \\
7 & 6 & $4 \pm 4$ & $15 \pm 4$ & $100 \pm 57$ & $23 \pm 8$ \\
\hline
\end{tabular}

For all the physicians, except for physician number 7 , the mean number of pieces of information provided by the system and the mean number of pieces of information searched by the physician are similar. This observation can be explained by several phenomena that we were able to observe:

- In some cases, although pieces of information provided were corresponding to what physicians needed, they asked their patients to confirm these pieces of information;

- In other cases, pieces of information provided were corresponding to what physicians needed, but they were not up to date and physicians had to search for updated information on their own. These cases occurred regularly during the clinical trials, particularly for pieces of information from laboratories external to the HCL, since the information was not available through Easily ${ }^{\circledR}$;

- In still other cases, pieces of information provided were corresponding to what physicians needed, but they also needed further pieces of information about the patient;

- Finally, in some other cases, pieces of information provided did not correspond to physicians' needs, and the latter therefore found themselves in a classical consultation situation.

Table II also shows that interactions with Easily ${ }^{\circledR}$, measured by the number of mouse clicks, differ greatly depending on the physician observed. Some physicians have developed work processes optimized according to their needs, while others follow more exploratory processes. Physicians who work in this second way are the ones who interact the most with Easily ${ }^{\circledR}$. Concerning the duration of the observed consultations, except for a few cases, they appear to be fairly stable from one consultation to another, because physicians tried to stay on schedule as much as possible.

To identify a general tendency concerning the impact of our system on the work processes of observed physicians, we have computed the correlation coefficients between the different criteria monitored. Pearson's correlation coefficient $\rho_{X, Y} \in[-1,1]$ of two variables $X$ and $Y$ is computed as detailed in (3). The closer $\rho_{X, Y}$ is to 1 , the more the variables $X$ and $Y$ are positively correlated. The closer $\rho_{X, Y}$ is to -1 , the more the variables $X$ and $Y$ are negatively correlated. And the closer $\rho_{X, Y}$ is to 0 , the less the two variables are correlated.

$$
\rho_{X, Y}=\frac{\mathbb{E}\left[\left(X-\mu_{X}\right)\left(Y-\mu_{Y}\right)\right]}{\sigma_{X} \sigma_{Y}}
$$

By computing these correlation coefficients for each pair of criteria, we obtained the correlation matrix presented in Fig. 4. The duration of consultation and the number of mouse clicks are highly correlated, with a correlation coefficient of 0.88 . To a lesser extent, the duration of a consultation is also positively correlated with the number of pieces of information searched by physicians. These two points make sense because the more a physician searches for pieces of information, or the more s/he needs to interact with Easily ${ }^{\circledR}$, the more the duration of the consultation is extended. On the other hand, the number of pieces of information provided by the decision support system seems to have a low degree of correlation with the duration of consultations, which validates our hypothesis concerning this point. The reason is that physicians try to stay on schedule as much as possible, with a fixed time for all consultations.

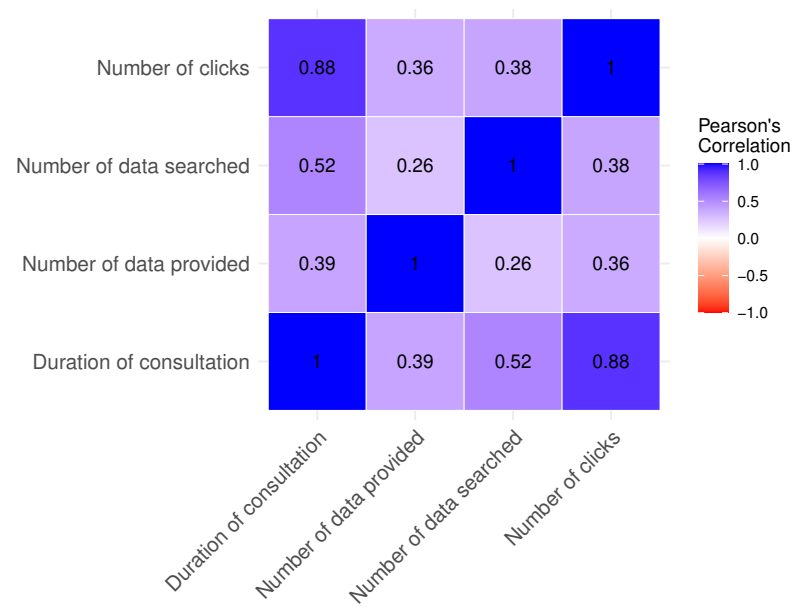

Fig. 4. Correlation matrix between each criterion observed during clinical trials of CoBoy 
The number of pieces of information provided by CoBoy has a low degree of correlation with the number of pieces of information searched by physicians and to the number of mouse clicks. Although the coefficients computed between these three criteria are not negative, they are relatively close to 0 . These results are in line with our hypotheses, because the rise of the number of pieces of information provided does not appear to have a high degree of correlation with the rise of the number of pieces of information searched by physicians, or with the rise of the number of mouse clicks. The support provided by CoBoy hence appears to have compensated for its entry cost.

Concerning the results of our questionnaire on the acceptability of our decision support system, they are positive. Fig. 5 shows the distribution of physicians' answers to the three main questions of our questionnaire, concerning respectively the useability of the system, its perceived utility, and the intention to use the system in practice. Because the five possible answers were different for the different questions, we have established a scale from -2 to +2 : -2 and -1 corresponding to negatives answers, 0 correspondings to the neutral answer, and +1 and +2 corresponding to positives answers. We have also decided to accept intermediate answers, because some physicians were not able to decide between two adjacent proposals.

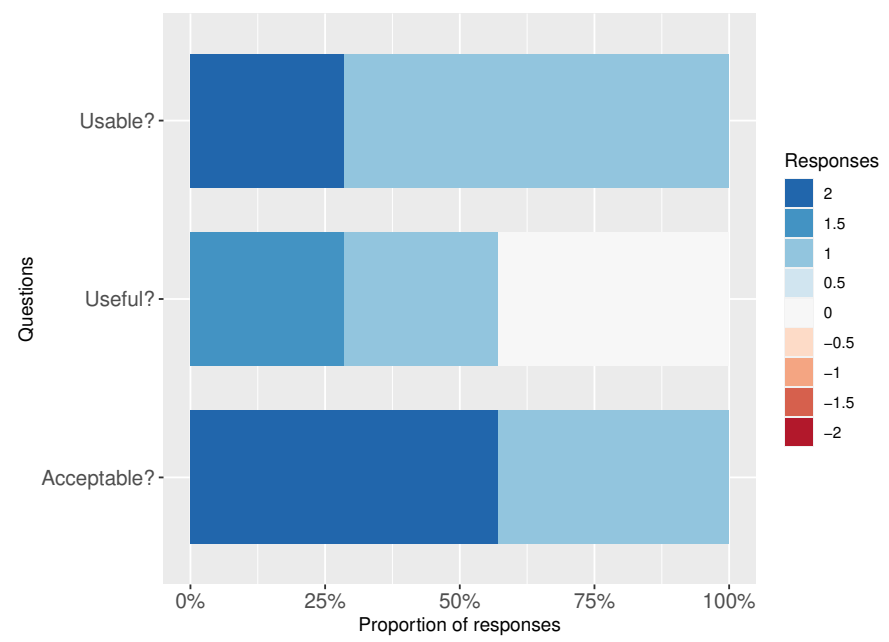

Fig. 5. Distribution of answers to the main questions of the questionnaire

$75 \%$ of physicians ( 5 physicians out of 7 ) found that getting started with CoBoy was "rather easy". The two remaining physicians found that getting started with CoBoy was "very easy". The inconvenience caused by the use of a second screen, but also by a fairly rudimentary user interface, were the main comments concerning the useability of CoBoy.

Concerning the perceived utility of the tool, results are more mixed but overall they are positive. More than half of the observed physicians (4 physicians out of 7) found CoBoy "rather useful" or "very useful". The other three physicians found CoBoy "Neither useful nor useless". According to comments made by this second group of physicians, they were not able to estimate the usefulness of the support system due to the limitations encountered during clinical trials, but they did not find the proposed approach "useless".

Lastly, concerning the intention to use CoBoy in practice, more than half the physicians (4 physicians out of 7) answered that they will "certainly" use it, if it is well integrated into Easily ${ }^{\circledR}$ and if the limitations encountered are overcome. The other three physicians answered "rather yes" to the same question under the same requirements. Despite the current limitations, the observed physicians were particularly enthusiastic about the possibilities offered by the decision support system. The involvement of physicians during the conception and development of the decision support system can have played a role in enhancing this high acceptability.

The most recurrent general comments were, for the most negative ones, about the current technical and ergonomic limitations of the system, and, for the most positive ones, about the working comfort offered by having access to a summary of information concerning their patient for the whole duration of the consultation. The suggested improvements were mainly about possible ways to have access to missing biological pieces of information, but also about various possible improvements for the user interface.

\section{LIMITATIONS AND PERSPECTIVES}

As explained previously, one of the main limitations to the viability of the support provided by our system is its impossibility to provide data coming from laboratories external to the HCL. However, the reasons for these limitations are not due to our decision support system but come from the context of the use of the system. Indeed, although large amounts of data on patients are available and accessible in the HCL's databases, if specific pieces of information necessary to take decisions are not available, the support provided by the system is crippled. A decision support system such as CoBoy must hence be built on a robust and viable structure.

Because our system is a prototype not integrated into the current work processes of physicians, it remains difficult to foresee its real impact in practical situations. Besides, the simple introduction of a second screen in the physicians' workspace is not without consequences, for two reasons. The first reason is that physicians are currently accustomed to using only one screen and having to navigate between two screens, or simply to think about looking at the second screen, can cause discomfort. The second reason is the physical space that a second screen takes in a consultation room which can create an additional physical barrier between the physician and her/his patient. However, some physicians noted the comfort allowed by the use of two screens, especially thanks to the possibility that it offers to record information on patients and write documents on one screen, while having access to a summary of information about the current patient on the other screen. An analysis of the physical space that computers take in a consultation room would be relevant to find a balance between comfort and physician-patient interactions.

Despite these limitations, the decision support system CoBoy have aroused interest among the physicians who have 
participated in the clinical trials. They articulated various suggestions to improve the decision support system and to overcome the problems encountered during the clinical trials. More specifically, physicians saw the potential utility of the system if these problems could be solved. Concerning the external biological analyses, for example, physicians proposed that these pieces of information should be recorded by themselves or their secretaries, before consultations or during consultations, using the user interface of Easily ${ }^{\circledR}$ or CoBoy. However, this could generate an additional workload. The relevance of engaging such an additional workload depends on the real usefulness of the support provided. The ideal situation would be to be able to extract, without risks of errors, results of biological analyses from reports of external laboratories, or to have a common structure between the HCL and external laboratories, allowing the communication of results to physicians.

Physicians also made various suggestions about the user interface of CoBoy. The main idea that emerged from discussions with physicians is that the user interface should provide, at a glance, a summary of the most important information on patients according to their diseases, and quick access to secondary information. The user interface proposed by [31], for the follow-up of patients with diabetes diseases, could be a basis for the future user interface of CoBoy, for all kinds of diseases. We also intend to give to physicians the possibility to indicate to the system which pieces of information to display under which conditions, through a system allowing physicians to configure the user interface to meet their needs. This could improve not only the working comfort for the physicians, but also their appreciation of the system. However, this could also generate an additional workload. Once again, a balance has to be found between the modularity of the user interface and this additional workload.

To summarize, although it is currently difficult to assess whether CoBoy impacts positively or negatively the work processes of physicians, the proposed approach is appreciated and accepted by the physicians who have participated in clinical trials. The many suggestions made by physicians for a better integration of CoBoy in their work processes highlight an interest in the proposed decision support system. The introduction of a decision support system such as CoBoy, aiming to provide physicians with a set of targeted pieces of information, appears to be adapted to the needs of physicians during customary medical consultations, and seems to be more acceptable than the approaches materialized by the current DDSSs [22]. However, other clinical trials should be performed once CoBoy will be integrated into Easily ${ }^{\circledR}$ and into the work processes of physicians, to have a more reliable assessment of its impact and its real acceptability.

Besides, we have developed the proposed system in the specific context of medical consultations concerning diseases treated in endocrinology. Although we aim to propose a support system as generic as possible, the proposed system might well fail to correspond to physicians' needs during consultations in other medical specialties. It would therefore be interesting to study, through other clinical trials, the applicability of the proposed decision support system in other hospital departments.

\section{CONCLUSION}

In this paper, we have presented a decision support system taking the form of a virtual assistant dedicated to supporting physicians during their day-to-day medical consultations. This system is the result of several years of works made in collaboration with the employees of the HCL on how to support physicians during customary situations such as medical consultations [22], [23], [26]. Our first goal was to propose a decision support system acceptable for physicians and adapted to the constraints and the challenges of supporting customary medical consultations.

Named "CoBoy", this decision support system can anticipate and provide pieces of information needed by physicians for their consultations, given common pieces of information on the patient: age, sex, BMI, and the disease for which s/he is followed-up. This system has been developed in collaboration with physicians working at the HCL's department of endocrinology. It is hence calibrated for diseases treated in this domain. Currently, CoBoy is able to treat around twenty distinct diseases and can search for, according to physicians' needs, among a hundred different pieces of information about patients.

We have conducted a set of clinical trials to evaluate the feasibility of the introduction of CoBoy in the work process of HCL's physicians. Although the impact of our system on physicians' work processes is not entirely assessable, our first results are positive. In addition, physicians who used CoBoy during these clinical trials showed a certain interest in it and showed an interest in being involved in the process of improving the decision support system. Although much work remains to be done, the decision support system we proposed corresponds to physicians' needs during medical consultations and to the constraints underlying decision support in such situations.

Further works are needed to evaluate more precisely the impact of such a decision support system on decision processes and physicians' workload. Besides, further works are also needed to propose a better user interface and to adapt our decision support system in several services of the HCL. However, the high acceptability showed by physicians during the clinical trials towards CoBoy, compared with the low acceptability of current DDSSs [19]-[21], supports the validity of our choice of a self-adaptive virtual assistant for supporting decisions during medical consultations.

\section{ACKNOWLEDGMENTS}

Works presented in this paper were made during the pandemic wave of SARS-CoV-2. This particularly impacted the clinical trials of the decision support system. We want to address a special thanks to Pr. Moulin, Pr. Raverot, Dr. Abeillon, Dr. Bouzehouane, Dr. Brac De La Perriere, Dr. 
Charriere, Dr. Lasolle, Dr. Moret, Dr. Renault, and Dr. VillarFimbel for their disposal and for allowing this work to be done. This paper is also the result of a long collaboration with the employees of the Civil Hospitals of Lyon (France). Thanks to all of them.

\section{REFERENCES}

[1] R. A. Miller, "Diagnostic decision support systems," in Clinical Decision Support Systems. Springer, 2016, pp. 181-208.

[2] J. M. Reider, "Impact of national policies on the use of clinical decision support," in Clinical Decision Support Systems. Springer, 2016, pp. $111-130$.

[3] E. H. Shortliffe, S. G. Axline, B. G. Buchanan, T. C. Merigan, and S. N. Cohen, "An artificial intelligence program to advise physicians regarding antimicrobial therapy," Computers and Biomedical Research, vol. 6, no. 6, pp. 544-560, 1973.

[4] E. Shortliffe, Computer-based medical consultations: MYCIN. Elsevier, 2012, vol. 2.

[5] R. A. Miller, M. A. McNeil, S. M. Challinor, F. E. Masarie Jr, and J. D. Myers, "The internist-1/quick medical reference projectstatus report," Western Journal of Medicine, vol. 145, no. 6, p. 816, 1986.

[6] R. A. Miller, "A history of the internist-1 and quick medical reference (qmr) computer-assisted diagnosis projects, with lessons learned," Yearbook of medical informatics, vol. 19, no. 01, pp. 121-136, 2010.

[7] V. Anand, P. G. Biondich, G. C. Liu, M. B. Rosenman, S. M. Downs et al., "Child health improvement through computer automation: the chica system." in Medinfo, 2004, pp. 187-191.

[8] D. J. Etter, A. McCord, F. Ouyang, A. L. Gilbert, R. L. Williams, J. A. Hall, W. Tu, S. M. Downs, and M. C. Aalsma, "Suicide screening in primary care: use of an electronic screener to assess suicidality and improve provider follow-up for adolescents," Journal of Adolescent Health, vol. 62, no. 2, pp. 191-197, 2018.

[9] S. M. Honaker, T. Dugan, A. Daftary, S. Davis, C. Saha, F. Baye, E. Freeman, and S. M. Downs, "Unexplained practice variation in primary care providers' concern for pediatric obstructive sleep apnea," Academic pediatrics, vol. 18, no. 4, pp. 418-424, 2018.

[10] A. Bernasconi, F. Crabbé, A. M. Adedeji, A. Bello, T. Schmitz, M. Landi, and R. Rossi, "Results from one-year use of an electronic clinical decision support system in a post-conflict context: An implementation research," PloS one, vol. 14, no. 12, 2019.

[11] C. Bessat, N. A. Zonon, and V. DAcremont, "Large-scale implementation of electronic integrated management of childhood illness (eimci) at the primary care level in burkina faso: a qualitative study on health worker perception of its medical content, usability and impact on antibiotic prescription and resistance," BMC public health, vol. 19, no. 1, p. 449, 2019.

[12] N. Asiri, M. Hussain, F. Al Adel, and N. Alzaidi, "Deep learning based computer-aided diagnosis systems for diabetic retinopathy: A survey," Artificial Intelligence in Medicine, 2019.

[13] J. De Fauw, J. R. Ledsam, B. Romera-Paredes, S. Nikolov, N. Tomasev, S. Blackwell, H. Askham, X. Glorot, B. ODonoghue, D. Visentin et al., "Clinically applicable deep learning for diagnosis and referral in retinal disease," Nature medicine, vol. 24, no. 9, pp. 1342-1350, 2018.

[14] K. Zhang, X. Liu, F. Liu, L. He, L. Zhang, Y. Yang, W. Li, S. Wang, L. Liu, Z. Liu et al., "An interpretable and expandable deep learning diagnostic system for multiple ocular diseases: qualitative study," Journal of medical Internet research, vol. 20, no. 11, p. e11144, 2018.

[15] A. Esteva, B. Kuprel, R. A. Novoa, J. Ko, S. M. Swetter, H. M. Blau, and S. Thrun, "Dermatologist-level classification of skin cancer with deep neural networks," Nature, vol. 542, no. 7639, pp. 115-118, 2017.

[16] T. C. Hollon, S. Lewis, B. Pandian, Y. S. Niknafs, M. R. Garrard, H. Garton, C. O. Maher, K. McFadden, M. Snuderl, A. P. Lieberman et al., "Rapid intraoperative diagnosis of pediatric brain tumors using stimulated raman histology," Cancer research, vol. 78, no. 1, pp. 278289,2018

[17] S. Joo, Y. S. Yang, W. K. Moon, and H. C. Kim, "Computer-aided diagnosis of solid breast nodules: use of an artificial neural network based on multiple sonographic features," IEEE transactions on medical imaging, vol. 23, no. 10, pp. 1292-1300, 2004.

[18] G. H. B. Miranda and J. C. Felipe, "Computer-aided diagnosis system based on fuzzy logic for breast cancer categorization," Computers in biology and medicine, vol. 64, pp. 334-346, 2015.
[19] D. F. Sittig, M. A. Krall, R. H. Dykstra, A. Russell, and H. L. Chin, "A survey of factors affecting clinician acceptance of clinical decision support," BMC medical informatics and decision making, vol. 6, no. 1, p. 6, 2006.

[20] T. Onega, E. J. A. Bowles, D. L. Miglioretti, P. A. Carney, B. M. Geller, B. C. Yankaskas, K. Kerlikowske, E. A. Sickles, and J. G. Elmore, "Radiologists' perceptions of computer aided detection versus double reading for mammography interpretation," Academic radiology, vol. 17, no. 10, pp. 1217-1226, 2010.

[21] R. Masud, M. Al-Rei, and C. Lokker, "Computer-aided detection for breast cancer screening in clinical settings: Scoping review," JMIR medical informatics, vol. 7, no. 3, p. e12660, 2019.

[22] A. Richard, B. Mayag, F. Talbot, A. Tsoukiàs, and Y. Meinard, "What does it mean to provide decision support to a responsible and competent expert?" EURO journal on decision processes, Aug. 2020. [Online]. Available: https://hal.archives-ouvertes.fr/hal-02916089

[23] A. Richard, B. Mayag, Y. Meinard, F. Talbot, and A. Tsoukiàs, "How AI could help physicians during their medical consultations: An analysis of physicians' decision process to develop efficient decision support systems for medical consultations," in PFIA 2018, Nancy, France, 2018.

[24] G. Tsoumakas and I. Katakis, "Multi-label classification: An overview," International Journal of Data Warehousing and Mining (IJDWM), vol. 3, no. 3, pp. 1-13, 2007.

[25] G. H. John and P. Langley, "Estimating continuous distributions in bayesian classifiers," in Eleventh Conference on Uncertainty in Artificial Intelligence. San Mateo: Morgan Kaufmann, 1995, pp. 338-345.

[26] A. Richard, B. Mayag, F. Talbot, A. Tsoukiàs, and Y. Meinard, "Transparency of classification systems for clinical decision support," in Information Processing and Management of Uncertainty in KnowledgeBased Systems, M.-J. Lesot, S. Vieira, M. Z. Reformat, J. P. Carvalho, A. Wilbik, B. Bouchon-Meunier, and R. R. Yager, Eds. Cham: Springer International Publishing, 2020, pp. 99-113.

[27] R. Sinha and K. Swearingen, "The role of transparency in recommender systems," in CHI'O2 extended abstracts on Human factors in computing systems. ACM, 2002, pp. 830-831.

[28] A. Holzinger, C. Biemann, C. S. Pattichis, and D. B. Kell, "What do we need to build explainable ai systems for the medical domain?" arXiv preprint arXiv:1712.09923, 2017.

[29] R. Caruana, Y. Lou, J. Gehrke, P. Koch, M. Sturm, and N. Elhadad, "Intelligible models for healthcare: Predicting pneumonia risk and hospital 30-day readmission," in Proceedings of the 21th ACM SIGKDD International Conference on Knowledge Discovery and Data Mining. ACM, 2015, pp. 1721-1730.

[30] R. Heeks, "Health information systems: Failure, success and improvisation," International journal of medical informatics, vol. 75, no. 2, pp. 125-137, 2006.

[31] A. Giordanengo, E. Årsand, A. Z. Woldaregay, M. Bradway, A. Grottland, G. Hartvigsen, C. Granja, T. Torsvik, and A. H. Hansen, "Design and prestudy assessment of a dashboard for presenting self-collected health data of patients with diabetes to clinicians: Iterative approach and qualitative case study," JMIR diabetes, vol. 4, no. 3, p. e14002, 2019. 